Discussion Paper No. 609

\title{
THE SOURCE OF SOME PARADOXES FROM SOCIAL CHOICE AND PROBABILITY
}

\author{
DONALD G SAARI*
}

Northwestern University

March 1984

(*) Department of Mathematics, Northwestern University, Evanston, IL 60201

Acknowledgements

A simple geometric argument is given which explains the reason certain paradoxes coming from the voting and the probability-statistics literatures occur. Particular attention is focused upon difficulties which occur with ranking methods and with conditional probability. The approach not only unites these paradoxes, but it also uncovers several new ones. 


\section{Introduction.}

The social choice literature is filled with deseripticns of "paradoxes". In this papier we explain why some of them occur; to do this, I will prouide an elenentary, geometric argument which describes the source of several of the paradoxes for the voting and the agenda manipulation literature. Of equal interest to economics is that this sane argument exposes nontransitive tehavior which arises in probability and statistics. This is of interest not only because it unites paradoxes coming from differert literatures, but also because it is comori for models in economics to be based upor probabilistic contepts such as conditional probability and the combination of random variatles. This is particularly true for the evalving field of information in ecoromics. Here it is quite commion to use constructs such as bayesian techniques and conditional probability. This is also true in other contexts where frices and other action variables are conditioned upon certain information. So, part of the thrust of this paper is to describe certain anomalies which crop up in these basic medelling concepts, (Sections 2 and 3), although our main emphasis will be with sociai choice types of examples.

Although the explanation given here is elementary, (it depends on the open mapping principle and some singularity theory), it appears to be new and it uncovers new examples. Indeed, one point which will emerge is the ease with which paradoxes can arise. This suggests that uniess farticular care is taken in models which use: say, conditional prabability, then there may exist consequences which are urexpected or undesired. Moreguer, the basic ides of the argunent given tiere can be abstracted in a form which will unite several conclusions from the social choice literature such as the Arrow Thearem, the Gibberd-satterthwaite Theorem, etc. This abstraction will be discussed elsewhere. To illustrate the argument, we will provide several new results concerning ranking procedures and voting. (Section 3.) 
A "paradox" is nothing more than a counter-intuitive behavior of a relationstip. Because this tehavior is unexpested, and perhaps undesired, it is important to know whether a class of paradoxes can be dismissed as being an anomaly consisting of isolated examples. Our argument indicates that this isn't the case; the type of examples which are discussed here recessarily accompany the modelling of a system and they do so in a robust fastion. This appraach will be used to determine tre informational cantent of these paradoxes--how prevalent are they and the type of analysis required to determine whether they do, or do not, occur. (section 4.)

To illustrate the flaver of these paradoxes, we start with two prototype examples. In the followirg sections I will indicate how thiey are related, and how they $c$ an be extended.

1. CONDITIONAL PROBABILITY AND SIMPSON'S PARADOX. Suppose a certain drug is to be tested in Chicago (C) and in Los Angles ( $C^{\prime}$ ). A test group (T) will receive the new drug, and a control greup (T') will receive the standard treatment. Sonie people are returned to health $(H)$, while others are not (H'). Assume that in both communities the new drug is determiried to be successful because it cures the sick withi a higher ratio than the standard treatment $\left\{F(H: C T)>P^{\prime}\left(H: C T^{\prime}\right), F\left(H: C^{\prime} T\right)>F^{\prime}\left(H: C^{\prime} T^{\prime}\right)\right\}$. Is it possible for the aggregated test results to have a reversed conclusion; namely, the new method is judged not to be successful because P(H:T)(F(H:T')? It is, and this is known as simpson's paradox. An explariation which differs from that given here? and an excellent discussion of this paradox and how it relates to the "sure thing" principle is given by $C$. Blyth in [1]. Examples where this paradox arises with real data car be found in [11].

It will be shown that this inconsistency phenomena is characteristic of conditional probability problems and models involuing the combination of random wariables. With the introduction of additional conditions, almost any imaginable 
extension can occur. For instance, suppose that the tests are conducted in university facilities (U) and in frivate labs (U'). There exist examples whereby the tests are unsuccessful in each university and in each lab as well as in each community, but then they are successful in the aggregate; or, there arist examples where the canclusions oscillate at each level in that the test is successful in each of the facilities, but they are unsuccessful in both communities, and then they are successful in the aggregate; etc.

Indeed, the appropriate ratio of euccess to failure in each of these examples can be made to exceed any predetermined constarit. For instance, with the above example where the canclusions ascillate, there exist situations where in each facility the probability of regaining fiealth by use of the drug is at least twice that obtained by use of the standard treatment. But when viewed from the vantage poirit of each community, the standard treatmerit is at least 3 times better than that achieved by the drug! Finally, when viewed from the total aggregated level, the drug is at least 4 times better thar the standard treatment!

2. VOTING AND RANKING METHODS: The issue of the aggregation of preferences is centrai to economics, where a common prototype system is voting methods. Here, a weil known phenomena can occur wher is:ra rate three alternatives $\{a, b, c\}$ with a weighted voting method. Suppose the outcome is abyc. It is possibie for a majority of the same voters to prefer $c$ to a, ever though this is the reversal of their original rankirig!

The situation can be much worse; this is indicated by the following example which illustrates the type of results we obtain. Assume given a ranking of Ny2 a) ternatives, say $a_{1}>a_{2}>\ldots>a_{N}$. If this represents a group's true linear ranking, then it would determine their pairwise rankings of the alternatives. To show that this need not be the case, designate, in an arbitrary fashion, a preferred alternative from each of the $N(N-1) / 2$ pairs of the alternatives. So, for each pair, 
this choice need not be consistent with the above ranking of the N alternatives, nor with the choice made for any other pair.

It will be shown that there exist examples of voters, each of which has a fixed, linear ranking of the $N$ alternatives, so that when the voters use the plurality voting system seach voter votes only for their first place alternative) to rank the iv alternatives, the result is the above $N$-fold ordering. On the other hand: when the same voters consider each of the N(N-1)/2 pairs, the alternative designated above will win with a majority vote. As a corollary, it folows that ali sorts of ranking paradoxes and agenda manipulation examples can be created. (The special case of this result where $N=3$ was given by $P$. Fishburn in [4]. A related class of voting paradoxes which also is subsumed by the analysis in this paper is given by 0.6 . Saari in [6,7],)

These examples illustrate that a true linear ordering may be impossible to achieve when individual linear rankings are aggregated, and they are characteristic of problems experienced by ranking methods for sets of random variables.

In this paper we will show how these paradoxes, as well as others, are related. For simplicity of exposition, we consider only those random variaties which have a finite number of values. However, because the explanation of the paradoxes is the open mapping principle, what follows extends in an obuious fastion to more general classes of random variables.

\section{Characteristic Functions and the General Result.}

The unifying explanation for all of the paradoxes discussed in this paper is the simple geometric property of open sets being mapped to open sets. The following standard statement, which ensures a "local open mapping", will be sufficient for most of what follows. 
Proposition 1. Let $F$ be a smooth mapping from a m dimerisional manifold $M$ to a $n$ dimensional manifold $N$ where $m \underline{n} n$. For some value $\underline{u}$ in the interior of $N$, assume there is a pre-image point of $\underline{u}$, say $\underline{p}$, which is an interior point of $M$. If the Jacobian of $F$ at $\mathrm{E}$ has maximal rank, then there is an open neighborhood of $\mathbf{Q}$ which is mapped onto an open neighborhood of $\underline{y}$.

For a more general setting where the domain and the range are function spaces, the Jacobian of $F$ is replaced with the Frechet derivative of $F$, and this derivative must satisfy the conditions far an open mapping.

We will use the prototype drug example to illustrate the basic ideas of this paper as to why the open mapping property captures the non-transitive behavior which is the source of the paradoxes. SLater in this section, this example will te used to motivate the statement of Theorem 3.) Following the proof, a general theorem for characteristic functions and conditional probability will be stated. Then, we conclude this section with the basic theorem for this paper.

Theorem 1. Consider Example 1 where a drug is compared with a standard treatment. Let $A$ be the variable representing the sets $C+C^{\prime}, C, C^{\prime}, C U, C U^{\prime}, C^{\prime} U$, and $C^{\prime} U^{\prime}$. For each of the 7 comparisons of $P(H: T A)$ with $P\left(H: T^{\prime} A\right)$, designate which probability is to be the larger. Indeed, for each $A$, choose a constant $d_{A}$ greater than unity and express the comparison as an inequality where the larger probability is to be at least $d_{0}$ times the smaller value. There exists examples which simultaneously satisfy all of the specified inequalities.

Proof. To prove the first part of the theorem, we need to show that for any choice of signs for the seven relationships P(H:TA)-P(H:T'A), there exist examples of data which will realize it. So, view these equations as being the 7 components of a mapping $F$ 
into $R$, where the domain is some space representing data points. The conclusion follows should the image set of the "comparison mapping" F meet all of the open orthants of R?.

Each of the orthants has a common boundary point, the origin 0. This "comparison point" will be used to prove the theorem in the following way. First, an interior point, $q$, af the domain is found in $F-1(Q)$ so that the Jacobian of $F$ at $Q$ has maximal rank. This implies that $F$ will map an open neighborhood of q onto an open rieighbarhood of $\underline{Q}$; such an open set must meet each of the orthants. Thus, for ary set of signs, there will be domain points, or examples, which will realize all of them simultaneously. So, the technical part of the proof is to express the domain and f in such a way so that $F$ is a smooth mapping.

There are 8 sets determined by the various intersections of the sets T, C, U and their complements. They are:

$S_{1}=T C U \quad S_{2}=T_{C}^{\prime} \quad S_{3}=T^{\prime} C^{\prime} \quad S_{4}=T^{\prime} U^{\prime}$

$S_{5}=T^{\prime} \mathrm{CU} \quad S_{6}=T^{\prime} \mathrm{CU} U^{\prime} \quad S_{7}=T^{\prime} C^{\prime} U \quad S_{8}=T^{\prime} C^{\prime} U^{\prime}$

An easy way to derive a smooth manifold $M$, which will serve as the damain of $F$, is to treat each of these sets as a disjoint space. Let xs designate the characteristic function of the set $H$ in $S_{s}$. Le: Ys represent the results at the community lewel;

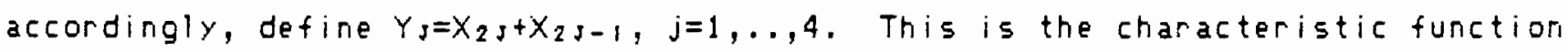

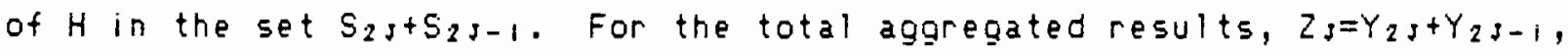
$j=1,2$, is the characteristic function of $H$ on the appropriate set.

If $x_{s}$ denotes the value of $P\left(X_{s}=1\right)$, then $x_{s}$ is in the unit interval 1 , $j=1, \ldots, 8$. Let $d s$ designate $P\left(S_{s}\right)$ in the space USk. The ds uariables describe a simplex in $R^{8}$ which is denoted by sik8) and defined by $\Sigma d_{s}=1$. Thus, these 16 variables 1 ie in the 15 dimensional space $M=1^{8} \times \operatorname{si}(8)$

By use of the standard relationship 2.1) $P(B)=P(B E)+P\left(B E^{\prime}\right)=P(E) P(B: E)+P\left(E^{\prime}\right) P\left(B: E^{\prime}\right)$, 
it follows that the probabilities $P\left(Y_{J}=1\right)$ and $P\left(Z_{J}=1\right)$ are rational functions of the $x^{\prime} s$ and the $d^{\prime} s$.

Comparison mag. The first four components of $F$ are given by

\section{2) $\{x, y-x j+4\}$ e}

where es is the unit vector in $R^{7}$ with unity in its jth comporent. This is the comparison of the results from the individual facilities. For comparisons at the community level $\left(A=C, C^{\prime}\right)$, the comporients of $F$ are

2.3) $\quad\left(y_{J}-y_{j}+2\right)^{\prime} t+4, j=1.2$ where

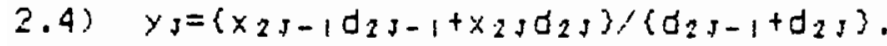

The function $y s$ represents $P\left(Y,=1: S_{2 s-1}+S_{2} s\right)$. The last component of $F$ corresponds to $A=C+C^{\prime}$, and it is

2.5) $\left\{z_{1}-z_{2}\right\} e ?$

where $z_{1}=\sum_{j=1}^{4} x_{j} d_{j} / \sum_{j=1}^{4} d_{j}$ and $z_{2}=\sum_{j=5}^{8} x_{j} d_{j} / \sum_{j=b^{\circ}}^{8} d_{j}$.

Oper mapping. Clearly $F$ is a smooth mapping. That the Jacobian of $F$ has maximal rank at some preimage of $\underline{\underline{0}}$ is a direct computation. Indeed, it follows from this

computation that the maximal rank condition holds everywhere except on a certain lower dimensional set in the preimage. SThese points of lower rank correspond to where either the value of the $y^{\prime} s$ or the $z^{\prime} s$ are determined because the corresponding pairs of $x^{\prime} s$ or $y^{\prime} s$ are equal.)

The specified choices of the 7 comparisons determines an or thant of R7; denote it by E. By construction, ali possible data points with this behavior are in $U=F-(\langle B\rangle$. By continuity, $U$ is an open set; we must show that it is nonempty. But, because $F$ is an open mapping in the neighborhood of some point in F-1(Q), (the Jacobiar tias maximal rank), F maps an open set from $M$ onto an open set of $\underline{0}$, and $s 0$ this open set meets $B$. Thus, $F^{-1}(B)$, and $U$, are nonempty.

Next, we must show that there are points in U which correspond to a "finite experiment". But, any rational point will suffice. A multiple of the common denominator of the $d s^{\prime} s$ is the total number of experiments. The same multiple of 
the numerator of $d s$ corresponds to the cardinality of $S \mathrm{~s}$, and it serves as a multiple of the denominator of $x_{J}$. Since the rational points are dense and since there is an open set of points which satisfy the conditions of the theorem, there are an infinite number of points which correspond to a finite experiment.

The set $F^{-1}(\underline{0})$. It remains to show that the inequalities can be bounded below by the designated constants. So, once the values of $d_{A}$ are specified, the inequalities are of the type $x_{j}>d_{A} x_{j}+4$ with a similar relationship for the y's and the $z$ 's. To see how the proof goes, let $q=(q, \ldots, q)$ be a point in this orthant. Then $F-1(q)$ is given by $x_{j}-x_{j+4}=q j$ with a similar relationship for the $y^{\prime} s$ and the 2's. If $q$ s is positive, then this becomes $X_{I} / X_{I}+4>1+Q I / X_{I}+4$.

If a point in $F^{-}($( $Q$ ) can be found where $x J+4$ is small enough so that the right hand side is bounded below by $d_{A}$, then the specified inequalities will follow. Thus, the mathematical explanation for these inequalities is that $F-1(\underline{0})$ contains $\underline{0} x s i(8)$ and that part of this set consists of boundary points for $U$. CThe $\underline{0}$ point permits the xs components to become arbitrarily small.)

A less abstract verification follows. Notice that $z J$ is between y $y^{\prime}$ and

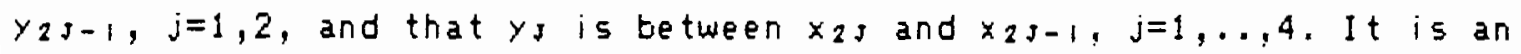
extension of the rank arqument that these are the only restrictions on these variables. Therefore, to allow for freedom in the choice of the $z$ 's, we require both $y_{1}$ and $y_{3}$ to be smaller than either $z$, and both $y_{2}$ and $y_{4}$ to be larger than either z. If all of these terms are sufficiently small, the smaller of each pair can be chosen so small that the ratio satisfies the appropriate inequality. Likewise, require both $x_{1}$ and $x_{5}$ to be smaller than either $y_{1}$ and $y_{3}$, while both $x_{2}$ and $x_{6}$ are to be larger. Also require both $x_{3}$ and $x_{4}$ to be smaller than either $y_{2}$ and $y_{4}$, while both $x_{4}$ and $x_{8}$ are to be larger. These inequalities are consistent with the ordering given above. Furthermore, the numbers can be chosen sufficiently small so that the ratio in each pair satisfies the required inequality. 
This completes the proof.

COMMENTS:1. The basic idea behind this paradox, and all of the other paradoxes described in this paper, is displayed by the comparision mapping $F$. Individual comparisons are one dimensional, but when more than one comparison is being made, they must not be viewed as being a series of comparisons on the line. More correctly, they must be viewed as defining a mapping where the range space is in a higher dimensional space. Such spaces admit symmetries and cycles, and so it should be expected that these cycles can be manifested in the various comparisons. This is what happens, and this is the source of the paradoxes! (In the example, the domain of $F$ is 15 dimensional while the range is only 7 dimensional. This suggests that this model admits added, simultaneous comparisons which lead to even more complex paradoxes; for example, they can be between different levels of aggregation.)

An easy way to demonstrate that all of these symmetries are available for a "comparison map" is to locate a point which is on the boundary for each of the comparison regions and to show that the image of the map includes an open set about this comparison point. Consequently, the range must intersect all of the comparison regions in non-empty open sets. For each of these open sets, the continuity of the comparison map provides an associated open set in the domain. If the admitted examples (e.g., in the above, the rational points) are dense in the domain, then in any open set there exist points which can be associated with examples. This simple idea is the essence of the explanation for all of the paradoxes given in this paper. Indeed, because the remainder of this section is devoted to refining this arqument, a reader primarily interested in the discussion of the ranking and the voting results can omit, on a first reading, the material from Theorem 2 to that just prior to Corollary 3.1.

2. A second fact emerges from this example. A careful investigation of the set F-1 (0) provides stronger conclusions. For instance, by using the boundary 
conditions of this set, the inequalities can be strengthened to be a ratio bounded below by any predetermined constant. A different issue might be to determine the minimum size of a sample set which is needed in order to obtain a particular behavior. For the above model, this would be given by a point in $U$ which has the smallest "lowest common denominator" after the admissible points are normalized as described above.

Other types of statements result from the structure of the range of F. For instance, the image contains an open set about the origin, so it meets any sector defined by a specified ratio of the outcomes; e.g., $\left[\left(P\left(Y_{1}=1\right)-P\left(Y_{3}=1\right)\right] 3\right) 42\left(P\left(X_{1}=1\right)-P\left(X_{4}=1\right)\right)>0$. The above shows that there are examples which will satisfy these conditions.

3. To avoid the above behavior, the Jacobian of $F$ can't be of maximai rank. Thus, this singularity constraint becomes a necessary condition to avoid a paradox. Often, as in the above model, these lower dimensional, singularity conditions correspond to familiar constraints such as the "independence of random variables". See Section 4.

The above demonstrates that "paradoxes" can te a common occurance; transitivity may be an unrealistically strong requirement. Nontransitive behavior should be suspected whenever the domain of the comparision map, $F$, is greater than 2 . In other words, unless proved otherwise, it is reasonable to expect that almost anything car. occur! This definitely is the case for problems in conditional probability. The same argument extends to concepts based upon conditional probability, such as indicators or prices which are based upon certain constraints. Moreover, the above demonstrates that when a paradox occurs, it may be associated with an open set of examples; accordingly, this type of behavior cannot be dismissed as being an isolated anomaly. Indeed, since finite examples correspond to rational points, and since when the denominators get larger (larger sample sets) more rational points are in any specified open set, such behavior cannot be minimized by expanding the sample size. 
The lead of Theorem 1 will be followed to obtain a gerieral theorem for a set of characteristic functions. To underscore the magnitude of the problem, we first determine the dimension of the domain for the associated comparison mapping. If there are $N$ characteristic furctions, then the first $N-1$ of them define $2^{N-1}$ possible sets. The last random variable can be treated as a characteristic function on each of these sets. In this way the comparison mapping will have a domain of dimension $2^{N-1}+\left\{2^{N-1}-1\right\}=2^{N-1}$. This number serves as the upper bound for the range (in order that the Proposition will apply), and it indicates that such a model admits a very large number of comparisons with possible concomitant unexpected behavior. Let $X_{s}$ be a random variatle with values in $S=\{0,1\}$ and with domain $D_{1}, j=1$, $\ldots, N$, where the D,'s are pairuise disioint. Let IN $=I \times \ldots \times I$, and let Si(N) be the usual simplex defined by the equation ..dk=1. Let the coordinates of the space $M=I N \times \operatorname{si}(N)$ be $\left(x !, \ldots, x_{N}, d_{1}, \ldots, d_{N}\right)$, where $x_{9}$ represents $P\left(x_{3}=1\right)$ and $d_{3}$ represents $P\left(D_{Y}\right)$ in the space $E=D_{1}+\ldots+D_{M}$. Let Y be a random variable with values in s which is defined by $f\left(x_{3}, \ldots, x_{n}\right)$. We assume that the domain of $Y$ is the union of those Do's with index j represented in function $f$. CHence $Y_{1}=f\left(X_{1}\right)=X_{1}$ has the domain $D_{1}$, while $Y_{2}=f\left(X_{1}, X_{2}\right)=X_{1}$ has the domain D!+D2, Then, it follows from 1.1 that $P(Y=1)$ is represented by a mapping from $M$ to the reg? line. This mapping is given by a summation where the terms are of the

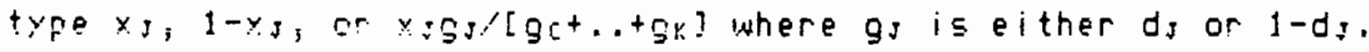

Theorem 2. Let $X_{J}, D_{i}, j=1, \ldots, N$ be as defined above. Let $Y_{J}$, with values in $S$, be defined in terns of some $k$-tuple of the $X^{\prime}$ 's with the convention an the domain as specified above. Assume that the admissible values for $P\left(X_{g}=1\right)$ and $P\left(D_{J}\right)$ form a dense set in $M$.

For s<aN pairs of these random variables, let ns be the mapping representing $P\left(Y_{I}=1\right)-P\left(Y_{H}=1\right)$ where $\left(Y_{I}, Y_{H}\right)$ is the $j$ th pair, $j=1, \ldots, s$. Define L:M=INXSi(N)--JRS as $\sum m s e j$. Let $q$ be an interior point of $M$ such that 
1) $q$ is in $L-1(\underline{Q})$, and

2) the Jacobian of L evaluated at q has maximal rank.

For each ordered pair, designate a preference between the wariables. Then, there exist examples where the randon variables assune all of these preference rankings simultaneously.

The discussion preceeding Theorem 2 provides a plausiblity argument why the above is true; the proof is in section 5. Of course, by examining the boundary conditions of the inverse image of L, it may be possible to show that the indicated ratios can be made arbitrarily large. This requires, as in Theorem 1, that the appropriate probabilities can be made arbitrarily small. Incidently, note that with a boundary analysis, Theorem 1 follows directly from Theorem 2.

The comparison mapping given above is determined by the differences between the probabilities of events. However since the paradoxes are explained by the open mapping principle, it is clear that this explanation extends to other situations. Indeed, all we need in order to define the comparison mapping is that the components can be expressed as smooth functions. So, the components could be functional combinations of probabilities, expected values, the various moments, waiting times, loss functions, decision rules, risk functions, correlation indices, scattering indices, variance, covariance, and on and on. If the open mapping principle applies at a comparison point, then all possible comparisons are realized. In this way, it is easy to show that there exist examples illustrating, for instance, that the waiting time for $A$ is larger than that for $B$, even though $A$ is the more probable event; that the expected value (or variance, loss function, etc.) may have $E(X)) E(Y)$, yet $E(f(X))(E(f(Y))$ for some monotonically increasing function $f$; or that certain decision rules may be inconsistent with other measures. CIndeed, a discrete version of this can be used to explain the Arrow social choice paradox.) Theorem 3 is the formal statement which covers all of these situations. 
In all of the above examples, the meaning of a comparision region and a comparision point are evident. In the next statement, their structural dependence is emphasized.

Definition. Suppose a mapping F:M- - SN is given. Comparison regions in the image space $N$ are sets of pairwise disjoint open sets of $N$. A comparison point is a point which is on the boundary of each of the comparison regions.

The statement of Theorem 2 suggests that the important comparison points for analyzing paradoxes are the regular points of $L$. This is not necessarily the case, the singular points of $L$ can be used to display an interesting and different class of "paradoxes". This can be easily seen with the drug example. Here, $\left.P\left(H: T C U^{\prime}\right)\left(P\left(H: T C U^{\prime}\right), P\left(H: T C^{\prime} U^{\prime}\right)\right\rangle P\left(H: T C^{\prime} U^{\prime}\right), P\left(H: T C^{\prime} U^{\prime}\right)\right) P\left(H: T C U^{\prime}\right)$, and $P(H: T C)\left\langle P^{\prime}\left(H: T C^{\prime}\right)\right.$ is an admitted outcome, but it cannot be captured by the above theorem. (The comparisor mapping is $\left.\left\{x_{1}-x_{2}, x_{3}-x_{4}, x_{2}-x_{4}, y_{1}-y_{2}\right\}_{2}\right)$ This is because the last inequality can not be reversed without interchanging others. But, the maximal rank condition on the comparison mapping means that it is possible to change in any inequality independently of any other inequality. Consequently, any setting which requires at least two inequalities to change signs simultaneously will necessarily correspond to a singular point of the associated comparison mapping. Mathematically, what is happening is that the graph of the comparison mapping is being pinched to form a fold or cusp at the comparison point. Consequently, the comparison point may not be an interior point of the image set of the mapping, but rather a boundary peint of the image. To capture the relationship among the comparison regions, the comparison point, and the domain, we use additional terms in the Taylor series expansion of the comparison map. After all, if the map is analytic or sufficiently smooth, then, locally, the image set is determined from this series. However, an analysis of a Taylor series can be very difficult. So, we provide 
alternative conditions in Theorem 3 which appear to be both sufficiently strong to handle the singularities which occur for a large class of models, and simplier to use because they reduce the number of terms which need to be evaluated.

Because the derivative conditions are mappings from tangent spaces, for simplicity assume that both the domain and the range of $F$ are affine spaces. This will allow us to blur the distinction between the spaces and their tangent spaces.

Theorem 3. Let $F$ be a smooth comparison mapping from a domain $M$ to the comparison space $N$ where the dimension of $M$ is bounded below by the dimension of $N$. Assume that the achissible values form a dense set in $M$. Let $c$ be a comparison value in $N$. Assume there is an interior point $p$ in the domain for which $F(p)=c$ and DFp has maximal rank. Then, the behavior characterized by any comparison region is admitted. Suppose that $B$ is a comparison region where its boundaries are given by hyperplanes passing through $c$. If any of the following are true, then there exist examples of the behavior characterized by $B$.

a) The range of DFp translated to $C$ meets $B$.

b) A combination of non-negative scalar multiples of elements from the range of DF, and the image set of D2F, translated to $C$ is in $B$.

Theorem 2 follows from the above because DFp meets all of the comparisori regions. The drug example, which was used to motivate the higher order derivative conditions, defines a mapping into $R^{4}$. It is a simple computation to show that this behavior is captured by statement b of this theorem.

The second part of this theorem becomes particularly important when the components of the comparison mapping are constructed from loss functions, variance, covariance, the nonlinear combination of random variables, etc. This is because the nonlinear nature of the modelling often leads to singularities at a comparison point. Incidently, this derivative condition can be extended to higher order terms in the way 
indicated in the proof. But, in practice, higher order derivatives are usually difficult to use because of the increased number of variables, so, alternative arguments which capture the above are preferred. Of course, in this theorem as before, the properties of the boundary behavior of the inuerse map can admit more striking examples.

In the following section, we will illustrate this theorem by discussing ranking methods. Another source of interesting paradoxes is $C$. Blyth's influencial paper [2]; his examples can be described in terms of Theorem 3. One of his paradoxes with random variables $X$ and $Y$ has $P(X\rangle Y)$ as close to unity as desired, even though $P(X\langle a)\langle P(Y<a)$ for all choices of a. This corresponds, of course, To the boundary behavior of the comparison mapping. (In his paper, the paradoxes are described in terms of examples. The above treatment explains and unites them, it shows that they can be extended in several ways, and it proves that the paradoxes are satisfied by open sets of examples. This last fact undercuts some of the critical comments about his conclusions which are in the articles immediately following [2].)

Note that the comparison regions are required to be open sets. Technically, this forces the existence of an open set in the domain of the comparison mapping. Then, the condition that the admissible examples are dense farces some of the admissibie examples to be in this open set. However, there are situations where we might want a comparison region which isn't an open set in order to capture "indifference", etc. The only difficulty for this setting is that, in general, the inverse image of a comparison region need not be an open set. So, if the conclusions are to hold, additional, stranger conditions must be imposed on the admissible points cor the modelling) in order to ensure that there are admissible examples in the inverse image set. An example how this is done is given in [6].

We conclude this section with some comments concerning when the converse holds. Of course, we are not as interested in knowing when the Jacobian is of maximal rank as we are in knowing when the existence of certain types of examples implies the 
existence of others. This is of importance because proving that a comparison map has maximal rank can be an extremely difficult, analytic argument. So, the value of a converse is if it tells us that the existence a finite number of certain types of examples implies the existence of all possible examples. This is because it would convert a difficult analytic proof into a computational one. o in examples cas oceur. Moreover, the existence of such examples may be possible to be verified by a computer search; hence the task is converted to one which can utilize current technology.

However, to prove a converse, we still run into difficult singularity problems. For example, the mapping $\left\{x^{\prime}, y^{\prime}\right\rangle$ maps $R^{2}$ onto $R^{2}$, but $\left\langle x^{7}, y^{8}\right\rangle$ doesn't, and they carlo distinquished by Theorem 3 because the singularity is of a sufficientiy high order. Therefore, in this partial converse, we avoid singularity problems by requiring the comparison map to be linear. Extensions will follow the same basic approach.

Carollary 3.1. Suppose that $F$ is a linear comparison mapping with range space RN. Fur thermore, assume that the $2^{N}$ or thants are the comparison regions. If there exist 2N-1+1 examples, each represented by a different comparison region, then $F$ is of maximal rank and all possible comparisons are adritted.

The proof is that the image of a linear space under a linear mapping is a linear. space. If the image includes points from $2^{N-1+1}$ or thants, then the image cannot be a $2^{N-1}$ dimensional space, but rather a $2^{N}$ dimensional space. The conclusion follows. Actually, we can get by with fewer examples and even in the same comparison regions as long as they cannot lie in the same plane of dimension $2 \mathrm{~N}-1$. Extensions to spaces with different types of comparison regions is much the same with the emphasis on the geometry; this is particularly of interest with the regions introdused in the next section with ranking and voting. 


\section{Ranking Paradoxes.}

In this section, we will use multivalued random variables to describe some of the implications of Theorem 3. A richer assortment of behavior is to be expected because the additional values force the comparison map to admit a larger effective domain. iTo see this, express such a random variable as a linear combination of characteristic functions, and then apply the dimension argument which precedes Thearem 2.) Because of the interest in the social choice, most of our illustrations of this behavior will be directed toward obtaining new results for the ranking literature, the voting literature, etc.

The first examples are from weighted, or positional voting. If a group is to rank $N$ alternatives, al,.., an, then a common method is to adopt scalar weights, wl, .., wn, which are not all equal and which will be used to reflect each voter's ordering of the $\mathrm{N}$ alternatives. Usualiy, the larger weights are used to indicate more favored alternatives; however, an inverted weighting system could be used where smaller weights are assigned to more favored alternatives. Whichever system is specified, each voter assigns the appropriate weights to reflect his ranking of the alternatives. In the obvious way, the group's ranking of the alternatives is determined by the sum of the weights cast.

The weights can be expressed as the components of a vector $W_{N}$ in $R^{N}$. In order to standarize this vectar and to indicate whether an inverted system is being used, we require $W_{N}$ to be the assignment of the weights for the ranking al>as? .. $a_{N}$. CThus, if the values of the components of $W_{M}$ are increasing, then $w_{N}$ corresponds to an inverted voting system.) For plurality voting, the weight vector is $(1,0, \ldots, 0)$. Another well known voting system, called the Borda Count, uses the weight vector $B_{N}=\{N, N-1, \ldots, 1)$. Call any weighted voting system where the weight vector is 
a linear combination of $B_{N}$ and $E_{N}=(1, \ldots, 1)$ a Borda voting system. (See [6].)

To describe the main result for voting systems, we introduce what we call a reverse neutral system. This is where the sum of the standardized vector and the vector assigned to the reversed ranking aN>aN-1).. >al is a multiple of $E_{N}$. $A$ Borda system always is "reverse neutral". An easy algebraic argument demonstrates that the space of reuerse neutral systems is a hyperplane in $R^{N}$ of dimension [iv/2] where [] is the "greatest integer function". A basis for this hyperplane can be computed directly. For example, for $N=3$, only the Borda systems are reverse neutral. For $N=4$, a basis for the hyperplane is given by $E_{4}, B_{4}$, and $(2,1,1,0)$. For $N=5$, a basis is $E_{5}, B_{5}$, and $(2,1,1,1,0)$; etc.

For $N$ alternatives, there are $N$ ! different possible categories of yoters as determined by the $N$ ! ways to linearly rank the $N$ alternatives. Since the sum assigned to any alternative is a linear relationship, the group's ranking of the alternatives is not altered should the vector sum be divided by the total number of voters. Thus, the domain for in s problem becomes (the rational points in) the simplex si(N!) which lies in the positive orthant of a $N$ ! dimensional space. So, if $A_{1}$ is the random variable assigned to alternative aI, then $P\left(A_{1}=w_{k}\right)$ reflects the fraction of voters which rank the iTH alternative in the KTH place. If $A$ is the vector valued random variable $\left(A_{1}, \ldots, A_{N}\right)$, then the group's ordering of the alternatives is determined by the linear ordering of the components of the expected value, $E(A)$.

Although this describes an important slass of commonly used voting methods, it is not clear how the conclusions of such a procedure should be interpreted. The following theorem, which includes Example 2, highlights this. (Example 2 is a specia) case because the weight vector $W_{N}=(1,0, \ldots, 0)$ is not reverse neutral for $\left.N>2.\right)$

Theorem 4. Assume given a set of $N>2$ alternatives, $A l_{N}=\left\{a_{1}, \ldots, a_{N}\right\}$. Rank the $N$ alternatives in an ordering RKN. Delete any one alternative and rank the resulting set $A l_{N-1}$ in a ranking $R K_{N-1}$. Continue this process of deleting an alternative 
and ranking the set $A l$, in a ranking Rk $\mathrm{s}$ for $j=3, \ldots, N$. The choice of these rankings can be arbitrary; they reed not be related in any way to each other.

Specify a weight vector WJ which is not reverse neutral to rank the alternatives in set $A 1 \mathrm{~g}, j=3, \ldots, N$. The choice of the weight vectors need not be related. Next, for each of the $N(N-1) / 2$ pairs of the alternatives, specify one of the pair as being preferred to the other. This specification can be independent of any of the rankings Rk, and of any of the other pairwise comparisons.

There exist examples of voters, each of which has a fixed linear ranking of the $N$ alternatives, such that when the same voters use the weight vector $w_{s}$ to order the alternatives in set $A l$, , the outcome is $R K_{1}, j=3, \ldots, N$, and for each of the $N(N-1) / 2$ pairs of alternatives, a majority of the same voters prefer the specified alternative.

A simple example of this thearem is that there exist situations where most of the voters prefer a to $b, b$ to $c$, and a to $c$, yet when these same voters vote on all three alternatives with a weighted voting system other than a borda system, the group outcome is $c>b>a$. This outcome is the reversal of the implied linear ordering given by "majority preference". A more striking example of inconsistency for NE is where a majority of the voters prefer as to ajti for $j=1, \ldots, 4$, as $a_{1}$ \{so, these alternatives form a cycle\}, $a_{4}>a_{1}$ for $j=1,2, a_{1}>a_{3}$, and $a_{j}>a_{5}$ for $j=2,3$. Still, by use of a plurality vote, these same voters rank the first three alternatives as $a_{1}>a_{3}>a_{2}$, the first four alternatives as $a_{2}>a_{3}>a_{1}>a_{4}$, and the total set as ay>a1>as>ag >a4. Other examples are limited only by the imagination of the designer.

There is a literature comparing the conclusions of pairwise majority voting with weighted voting methods. (For example, see the expository article [5].) An alternative is called a Condorcet winner if it wins a majority vote against all of the other alternatives. Because a Condorcet winner does not always exist $4.9 .$, the 
above, second example), schemes have been proposed to determine the winning alternative based upon formulae which use the ordinal outcome of each of these Dairwise comparisons. For instance, the winning alternative could be one which wins the largest number of pairwise elections. Another possibility would be to select a (say, the status quo) if it is the Condorcet winner; otherwise, from the set of alternatives which beat al, select the one which wins the most pairwise comparisons. Call any such method a Condorcet Method $[5,8]$. An implication of Theorem 4 is if $N>3$, then for any Condorcet Method and for any weighted voting method which isn't reverse neutral, there exist examples of voters such that the outcomes will be as inconsisterit as desired! For instance, the winner tased upon the condorcet Method could be the last place alternative in the ranking which is determined by the weighted voting method.

Corollary 4.1. Assune there are $N \geq 3$ alternatives which are to be ranked first by a given Condorcet method and then by a weighted voting method which is given by weight vector $W_{N}$. Assume that $W_{N}$ is not reverse neutral. Let RKN be any rarking of the $\mathrm{N}$ alternatives, and let as be one of the alternatives. There exist examples of vaters such that the group's selection according to the Condorcet method is as, but the ranking of the $N$ alternatives as given by the weight vector $W_{N}$ is $R K_{N}$.

The choices of as and RKN are independent of each other, so the corollary displays the inconsistency of the outcomes. Of course, Theorem 4 asserts that this same chaotic state of affairs persists at all levels; indeed, it cannot te eliminated even if an assignment method combines, in some way, all of the ordinal wk outcomes at the $k=3, \ldots, N$ levels. For example, we could call a process "dynamic" if it uses the ranking of $\mathrm{N}$ alternatives to eliminate an alternative, if it uses the ranking of $N-1$ alternatives to eliminate another alternative, etc., until only one alternative remains. A dynamic and a Condorcet method need not agree. 
Corollary 4.2. Let $f$ be a dynamic method used on the set of $N$ alternatives to determine one alternative. Assume that for the set of $j$ alternatives, the voting vector $W_{3}$, which isn't reverse neutral, is used to rank the alternatives. Then, for any two alternatives as and $a x$, there exist examples of voters so that the result of the dynamic process is ax and the result of the specified Condorcet method is as.

Recently there has been interest in election procedures where the voter can choose among several different voting vectors $W_{N}$. One type is the bullet vote where a vater can use either the $5 y s t e m(2,0,0, \ldots, 0)$ or the $\operatorname{system}(1,1,0, \ldots, 0)$. In general, the voter can either split his vote among a specified number of his top ranked alternatives, or he can cast the full vote for his top ranked alternative. Another procedure, which is teing championed by S. Brams and P. Fishburn, is approval voting. Here a voter votes either approval or disapproval for each alternative. This means that this voter has $N$ different choices of voting systems to choose from: $(1,0,0, \ldots, 0),(1,1,0, \ldots, 0), \ldots,(1,1, \ldots, 1)$. So, in general let $\boldsymbol{W}^{N}$ denote the set of voting methods from which the voter can select to rank $N$ alternatives.

Corollary 4.3. Assume N/2 alternatives are given. Let the set $A l s$, and the ranking RKJ be as specified in Theorem 4 for $j=3, \ldots, N$. For each choice of $j$, let be a set of voting methods which the voters can use to rank the $j$ alternatives where each set contains at least one method which isn't reverse neutral. Select a Condorcet method, and select some one alternative ax. Then, there exist examples of voters such that when the same voters rank Als by the multiple voting method $\mathbf{W}^{\mathbf{f}}$, the outcome is $R K_{J}, j=3, \ldots, N$, while outcome for the Condorcet method is ak. 
based upon approval voting is the Condorcet winner; there exist examples where the results based upon approval voting are $\left.a_{4}\right)_{a_{3}} \mathbf{z}_{2}>_{a_{1}}$, for a set of 4 alternatives, al $a_{2}>a j$ for this set of three alternatives, and az is a Candarcet winner.

Related inconsistency results hold for if reverse neutral weight vectors are used. However, in this setting, the image of the comparison mapping may be a hyperplane rather than an open set. Thus, the choice of the rankings at various levels and the winner of the condorcet method may share some relationship; yet they need not be consistent. The types of results depend upon the dimension and the properties of the image of the comparison mapping.

The proof and an extension of Theorem 4 is given in section 5 , but it should be clear that it will involue a comparison mapping from $5 i(N !)$ to a Euclidean space of dimension $N+(N-1)+\ldots+3+\{(N)(N-1) / 2\}$. The first $N$ components of this mapping are given by $E(\underline{A})$, the next $N-1$ by the expected value of the we ighted voting method $W_{N-1}$, etc. The 1 ast $N(N-1) / 2$ components are given by the pairs $P\left(A_{I}\right)-P\left(A_{S}\right)$. Because the domain was normalized to Si(N), the image of the expected value for Ws lies in the simplex $S i(j)$ in $R^{J}$ where the sum of the components equals the sum of the components of Ws. Without loss of generality, we can assume that this sum is unity. Thus, the range of the comparison mapping is $\operatorname{Si}(N) \times S i(N-1) \times \ldots \times S(3) \times J N(N-11 / 2$ where $J$ is the interval $[-1,1]$. The range space has dimension $N^{2}-N-1$. The comparison value on each simplex is the point of complete indifference, $N^{-1} E_{N}$. For each of the intervals J, the comparision value is 0 . Thus, this is an example where both the domain and range are manifolds different from a Euclidean space, and the critical value for the mapping is not $\underline{0}$; but rather the indifference points $\left(N-1 E_{N},(N-1)-1 E_{N}-1, \ldots, 3-1 E_{3}, 0,0, \ldots, 0\right)$.

If $N>2$, then the dimension of the domain either equals that of the range (NF3), or it exceeds it $(N\rangle 3)$. (In the latter case, additional comparisons can be included.) The proof consists of showing that this linear map has maximal rank. The rank 
condition doesn't hold if $w_{k}, k=3, \ldots, N$ are Borda vectors. Here it turns out instead of being of maximal rank (i.e., corank 0), the linear map has a corank (with respect to the image space) of $(N(N-1) / 2\}-1$. This means that while there are several possible inconsistency statements, rot all of the arrangements are possible. In particular, Theorem 4 does not hold if the Wh's are Borda vectors. A direct verification of this for $N=3$ is given in section 5. For other reverse neutral systems, the corank dimensions are smaller, so more "inconsistency" statements are admitted. Since the Borda weight vectors avoid the largest number of these paradoxes, this serves as one argument for its adoption. By use of different techniques, P. Fishburn [4] proved Theorem 4 for the special case $N=3$. (Actually, what Fishburn proved is that the first example which immediately follows the statement of Theoren 4 can occur; but his proof probably could be extended to our general statement for $N=3$.$) For N>3$, the part of the theorem without all the possible pairwise comparisons is a special case of a result in [7].

Incidently, rote that the comparison mapping is linear, so $L-1(\underline{Q})$ must be a linear subspace which has dimension at least $N !-N 3+N$. Therefore, should $N>3$, then this subspace must intersect the boundary of the domain Si(N!). This implies that there exist several strong "boundary" examples of the type derived for simpson's paradox. Indeed, a boundary point in this model requires several of the comporients to be zero, so there exist examples where all voters (not just a majority) prefer certain alteriatives over others. An example illustrating this boundary behavior is given in Corollary 4.5 .

The above identifies the source of problems experienced in the social choice literature. The natural domain for considering $N$ alternatives is Si(N!); it has dimerision unity only when $N=2$, and then it increases rapidly for $N>2$. Whenever this large dimensional domain is exploited by defining a comparison mapping where the indifference point is an interiar point of the image set, then all sorts of behavior are admitted. For instance, it can be seen from the large dimension of the kernel of 
the comparison mapping in Theorem 4 that several additional comparisons can combined with the above to create an even more impressive inconsistency paradox. One extension of this type is offered in Section 5. What we show is that not only can there be inconsistency in results between levels, but if the voting methods satisfy certain technical conditions, then there can also be inconsistency when the same voters use different methods to rank the same set of alternatives. Even after this extension: the kernel has a high dimension, so several other types of examples are possible andor striking "bourdary" behavior examples can be constructed.

These ranking paradoxes are not restricted to voting and social choice. Similar difficulties accompany ranking procedures coming from probability and statistics, and they rely upon the second half of Theorem 4. To be underscore its importance, it will be restated below. Notice that it asserts that any type of cycle and subcycle can occur.

Corollary 4.4. Assune given $N>2$ alternatives $31, \ldots, a_{N}$. For each of the $N(N-1) / 2$ ordered pairs $(21,2 y)$, designate one alternative as preferred to the other. Then there exist examples of voters' preferences leach voter has a fixed linear ordering of the $N$ alternatives) such that for each of the $N(N-1) / 2$ ordered pairs, a majority of the same voters have the indicated preference.

To see how this corollary describes ranking procedures other than voting: suppose we are to determine a "quality ranking" of $N$ firms which make the same product. The "iTh alternative" now becomes the iTH firm, the "jTh voter" becomes the "jTH vector sample" of the product taken from each of the $N$ firms, and the "jth voter's preference ranking" becomes the linear "quality ranking" of the products in the jTH sample. The statement that as las has the interpretation that based upon the samples, firm i's product appears to be superior to firm j's. However, because there is an open set of examples such that all possitle pairwise comparisons are admitted, 
it follows that the binary sampling approach need not lead to a linear ordering of the "quality of the firms".

Indeed, thie well-known steinhaus paradox [9], where $\left.\left.a_{1}\right\rangle_{2}, a_{2}\right\rangle_{3}$, but ay $a_{1}$ for three firms producing the same product, is a special case of Corollary 4.2. Incidently, Theorem 4 admits a similar interpretation, so even should the firms be ranked by use of weighted ranking methods, the results still could be difficult to interpret. For instance, the weight vectors $w_{k}=(1,0, \ldots, 0)$ correspond to the natural ranking method based upon $P\left(X_{I}=m a x\left(x_{s}: j e A_{k}\right)\right)$. If these weighted ranking methods are used, then it follows from Theorem 4 that should some one firm be deleted, the revised ranking could drastically change. Other measures experience similar problems.

A similar effect occurs for the scoring of athletic events where a voter's preference corresponds to how the various teams placed in a particular event, etc. As another examile, for a sophisticated individual whose taste preferences are based upon several attributes (e.g., a wine connoisseur), we should not expect his binary comparisons to necessarily be transitive. This is, of course, an $N$ alternative version of the famous folklore "pie" example. (I prefer "apple" to "cherry". But, if "blueberry" is also available, then my choice is "cherry".)

As one might expect, this corollary has important implications concerning methods commonly employed to find the "best" choice from a field of alternatives. Indeed, it can be used to indicate the difficulties which can arise when tree diagrams and other hierarchical processes based upon some measure are used to determine the "best" alternative. This is because the corollary asserts that any path, or cycle amorig the alternatives is possible. Corollary 4.4 will be used to indicate that those nis s.t.s based upon pairwise comparisons and tree diagrams cari iead to any type of conclusion; thus the final outcome may be more a function of the initial "seeding" than the other variables. This includes such methods as tournaments, whether single, double, or $k$-fold elimination, the construction of agendae for meetings, etc. The following 
corollary, which illustrates this, asserts that the right to set an agenda for a meeting is a potential source of power (the first conclusion) or embarassment (the second conclusion where the final nutcome need not reflect, in any way, the true sentiment of anyone in the group). Similar evamples can be constructed for yarious tournament frocedures and other tree diagrams which are based upon pairwise comparisons. Equiualent examples for other hierarchical schemes which are based upon other measures (rather than the simple counting or frequency measure used here) can be constructed in much the same uav.

Corollary 4.5. Let N) 3 alternatives be given. An agenda is an ordered listing of the $N$ alternatives. The first two listed alternatives are voted upon; the alternative receiving the majority yote is then compared with the third listed alternative. This pairwise comparison procedure is continued to the end of the listing, and the alternative receiving the majority uote in the last comparison is the group's accepted a! ternative.

There exist examples of voters and $N$ agendae such that when the same voters use the $j$ TH agenda, the outcome is as, $j=1, \ldots, N$.

Indeed, if $N) 3$, there exist examples of voters and $N$ agendae so that the above conclusion holds even though all of the voters prefer a 3 to a4, a4 to as, .., and $a_{N-1}$ to $a_{N}$.

As in section 2, there exist open sets in the domain which exhibit each af the above behaviors. Consequently, these examples cannot be dismissed as being isolated because they are manifested by an infinite number of examples; the behavior is robust. As the number of agents increase cthe denominators of the rational points become larger), so do the number of the possible examples. Indeed, 
4. Let $n(\theta, m)$ be the percentage of possible outcomes which have the behavior $Q$ when there are magents. If the characteristics of the voters is uniformly distributed, then as $m-->\infty, n(Q, m)$ approaches the ratio of the area of $L-1(Q)$ to the area of the simplex.

For elementary number theoretic reasons, the sequence $\{n(Q, m)\}$ need nat be monotone. The limit is positive if $L-I(Q)$ contains an open set. For other distributions, the ratio is determined in a similar fashion, but with the different measure.

The atoue demonstrates the perils of weighted voting methods. It should be expected that other techniques which lead to a comparison mapping with a high dimensional range, don't fare much better. For instance, consider a dice game where each player uses his own die which is marked in the standard fashion. In a game of high score wins, suppose the loser pays the winner the difference between the face values. It is possible to weight the dice so that the expected payoff from the first die is more than the second, even though the second die has a higher probatility of winning in any roil of the dice. Indeed, suppose there are three different payoffs where the first is the cube of the difference in face values, the second is the difference of the squares of the face values, while the third is the difference of face values. For each payoff, select which die which is to have a larger expected payoff, and select which die is to have the better probability of winning a roll. It is possitle to weight the dice so that all four conditions can be satisfied simultaneously. This illustrates the possible incompatibility among various reward functions ard the distribution. Cof course, the result follows because of the functional independence of the form of the payoffs and the computations of probabilities.) The relationship of this type of example to models in economic theory is abvious. 


\section{Some Comments}

Recause the situations described above can be realized by open sets of examples. they cannot be dismissed as being anomalies. Instead, the lack of trarsitivity and the existence ef the various types of cyclic behavior should be viewed as being a natural phenomena. For those models where a preference relationship has a natural definition, such as in a dice game, this means we must accept the existence of this behavior as a fact. Since what corresponds to an "optimal situation" can change with the setting, then so must any strategy which is geared toward "rational behavior" or. an optimal outcome. For instance, in the dice example, we know which die we would prefer--it is the one with which we would get the largest payoff, Carsequently, if the functional form of the paroff changes, or if other dice are admitted las is to be expected, sets of weighted dice can create cyclic examples of the flavor given in the voting paradoxes), then what constitutes the "best choice" may varies accordingly without any regard for transitivity.

While this randomness in the behavior may be probable, ofter in the modelling of economics, the goal is to achieve some sort of a linear ordering of the alternatives. So, we need conditions which ensure that this will occur. There are two natural ways to do this--the first is by restricting the choice of the random variabies and the second is by restricting the space of admissible examples. The first approach is to determine those conditions on the comparison mapping which will avoid the undesired behavior. This is done by determining the conditions on the random variables so that the various derivative conditions in the Taylor series will not admit a paradox. An analysis of this type leads to a generalized form of "independence of the random variables". Indeed, an example which illustrates this is the definition of independence of random variables. It can be viewed as resulting from a singularity analysis for the comparison mapping with components of the form 
$P(X=i) P(Y=j)-P(X=i, Y=j)$. The derivative conditions force the components of this mapping to te identially equal to zero. But note, such severe singularity conditions aren't always required; fold conditions, as captured by Theorem 3 , are more general and they can obtain the desired results. For instance, certain equalities can te replaced with certain inequalities.

In order to ottain a linear relationship, it is natural to try to define a binary relationship which is sufficiently strong so that it will exclude nontransitive Eehavior. In my opinian, this is the wrong approach because the resulting binary relationship tends to be so severe and restrictive that it becomes impractical. For instance, for the voting examples with $N$ alternatives, a binary relationship with this property is where $a_{I}$ >a iff in the pairwise vote, alternative i received at least $(N-1) / N$ of all of the vates. (See $[3,10]$.$) This condition is unlikely to be satisfied$ in actual elections. A similar condition occurs for sampling, and again this creates an impractical, overly stringent condition.

A more reasonable approach for modelling would be to define a relationship in terms of what is intended. For instance, suppose a relatianship is to be defined with the purpose of firding a linear ordering of a set of it alternatives. As showri above, this shouldn't be cone in terms of a binary relationship. Therefore, the relationship should be defined to require the linear ordering to hold over all subsets of a) ternatives. For instance, if $N=4$, then a linear order is attained only if the order is preserved by the 6 possibie pairwise comparisons, the 4 triplet comparisons, and the comparison of the set of 4 alternatives. So, the definition will involve 11 relationstips. SEven here, without imposing additional conditions, it car be asserted that this is a linear ranking only with respect to the adopted ranking techniques. This is because there exist examples where the rankings at each level can change with the ranking technique. See [7], or the extension of Theorem 4 in Section 5.) Although this imposes a large number of conditions to check, they determine the largest possible sample space which defines a "true"linear ordering of the 
alternatives. Any other definition which leads to such an ordering must eliminate any legitimate admissible sample point which has an image close enough to the comparison point so that a small perturbation could cause a paradox.

Notice that these conditions are similar in spirit to those used in the definition of independence for a set of $n$ random variables; the independence coriditions must hold not only with binary comparisons, but also for all k-fold comparisons for $k=2, \ldots, n$. The reason is the same; lower level comparisons are not sufficient to avoid undesired behavior admitted by the comparison mapping which involves larger sets.

If the purpose of a relationship is anly to determine the "best" alternative, then the above conditions can be relaxed. The definition of what is "best" affects the definitian of the relationship. If it is to be the "best" not only at a pairwise comparison level, but also at a level with triplets, ete., then this reflects the conditions of the definition. For instance, if the alternative is selected by voting and it is meant to be the best over all other alternatives, then the concept of a Condorcet winner must be extended so that the alternative is not only the winner in all pairwise elections, but it is also the top alternative in all triples, etc. But, by adding restrictions in a definition, the size of the set in the domain which satisfies all of them is decreased. In turn, this increases the probability that the resulting definition will not be satisfied. Thus there is a tension between what is desired of a relationship and whether it is likely to be violated.

This approach can be further illustrated by considering the definition of a binary relationship which is intended to capture a certain property. Typically, such a relationship is tacitly assumed to be invariant over a certain class. However, as it was illustrated above, this need not be the case. For instance, it is a direct application of Theorem 3 to show the existence of a monotone function f and random variables $X, Y, Z$, and $W$ so that conditions of the type $E(X) \geq E(Y)$, but $E(f(X))(E(f(Y))$ occur, or that $\operatorname{Covar}(X, Z)>\operatorname{Covar}(Y, W)$ but $\operatorname{Covar}(f(X), f(Z))(\operatorname{Covar}(f(Y), f(W))$. This is 
true for almost any type of problem; for loss functions, for higher order moments, etc. If one of these measures is to be used to produce a binary ranking, then it runs the embarassment that a scaled version of the same random variables doesn't satisfy the same ranking. So, to handle this problem, the binary relationship must be redefined so that intended behavior of invariance over a certain class does occur. (This is a common approach for certain of these measures.) Namely, we would define a binary relationship to state that $X>Y$ iff the appropriate inequality holds not only for the random variables $X$ and $Y$, but $a l s 0$ for $f(X)$ and $f(Y)$ where $f$ a furictian fram the intended invariance class. (Note that if $X$ and $Y$ are finite valued, ther: only a finite choice of the f's need be checked to determine whether the conditian holds,?

A second way ta derive conditions which will avoid "undesired behavior" is to determine the appropriate conditions on the distributions of admissible examples. That is, restrict the domain of the comparison mapping. In theory, this is easy to do; the apprapriate regions are defined by $L-1(Q)$ where $Q$ carresponds to the comparison regions of a desired behavior. The characterization of these regions leads to conditions similar to the well known "Black's single peakedness" conditions of social chaice. However, with the exception of certain simple systems where an elementary analysis of the inverse image of a comparison point is possible re.g., see [7]), this characterization problem appears to be difficult.

Finally, a related approach is to determine whether it is likely that one af the above domain conditions are satisfiedi i.e., that a linear ordering is satisfied or that the "best" choice was made over a specified domain. This involues defining a relationship as described above, and then developing a standard testing procedure which is based upon the null hypothesis and the adoption of the appropriate significance levels. This again depends upon the analysis of the sets $L-1(Q)$, and it is a task which remains open for most systems. 


\section{Proofs}

In this concluding section, the proofs of the farmal statements will be given. The key result is Theorem 3 . This statement includes Theorem 2 as a special case.

Proof of Theorem 3. To prove the theorem, two facts are needed. The first is that the image set of $F$ meets the appropriate comparison regions. The second is that if $Q$ is ane of the specified camparison regians, then an admissible example point is in the set $F-1(Q)$. However, the comparison regions are open subsets of the range space. Therefore, if the image of $F$ meets $Q$ at a point $Q$, then the set $F-1(Q)$ is a nonempty open set. The admissible points are assumed to be dense, so there will exist admissible points in $F-1(Q)$.

What remains is to show that the conditions ensure that the image of $F$ will meet the appropriate comparison regions. The first condition ensures that an open neighborhood about $p$ will be mapped onto an open set about $c$. Since $c$ is a boundary point for each of the comparison regions, this open set has a nonempty intersection with each of the comparison regions.

To show how the remaining candition ensures that $F$ meets $B$, let a(t) be a smocth curve in the domain for which $a(0)=p$. We will show that the curve can be selected so that if the conditions of the Theorem are satisfied, then $F(a) i)$ is in $B$ for a sufficiently small value of $t$.

According to the chain rule,

$5.1 F(a(t))=c+D F_{P}\left(a^{\prime}(0)\right) t+(1 / 2)\left[D z F_{P}\left(a^{\prime}(0), a^{\prime}(0)\right)+D F_{p}\left(a^{\prime \prime}(0)\right)\right] t z+0(t z)$.

The last term designates terms which, when divided by $t 2$, approach zero as $t-->0$, If the first condition is satisfied, then choose $a^{\prime}(0)$ so that $D F_{p}\left(a^{\prime}(0)\right)$ is in $B$. Since the comparision regian $B$ is wedge-like in a neighborhood of $C$, this conclusion holds for any positive scalar multiple of this term. But, for a sufficiently small value of $t, F(a(t))=c+t D F p\left(a^{\prime}(0)\right)+o(t)$. Because the first two terms on the right 
hand side define an interior point of $B$, so does the right hand side for sufficiently small values of $t$. This completes the proot.

Now suppose that the range of of does not meet $B$, but a combination with nori-negative scalar coefficients of terms from the ramge of DF and the range of D2F, when restricted to the kerriel of DF, meets $B$. This means that there is a choice of $a^{\prime}(0)$ in the kernel of DF ard a" (0) such that the bracketed term meets $E, \vdots$ itce $\therefore=$ $D F\left(a^{\prime}(0)\right)$ term is zero, the argument essentially is the same as the one given atove.

To prove Theorem 4, we first prove the Carollary 4.4 . Procf of corollary 4.4. For each of the pairs of alternatives, a voter wauld vote either 1 or -1 to indicate the preferred alternative. More specifically, list the pairs in the following order. The first pair is (a1, az); the second set of two pairs is given ty $\left(a_{3}, a_{3}\right\rangle, j=1,2$; and the kth set of $k$ pairs is given ty $\left(a j, a_{k+1}\right\rangle, j=1, \ldots, k ; k=3, \ldots, N-1$. For each pair, the voter assigns the weight 1 if the voter prefers the first listed alternative over the second one; otherwise the voter casts the weight -1 . For example, associated with the preference ranking $a_{1}>a_{2}>\ldots>a n$ is the vector with unity in all $N(N-1) / 2$ of the components.

Since the $N$ alternatives can be ranked in N! different ways, the comparison map is a linear mapping from $S i(N)$ to $J N(N-1) 2$ where $J$ is the interval $[-1,1)$ and the comparisori value is $\underline{0}$. We must demonstrate that there is an interior point iri si(N)! which is the preimage of $\underline{Q}$ and that the Jacobian of the comparison maf has full rark at this point. Such an interior point is obtained by having an equal number of voters for each of the N! possible ways to rank the alternatives.

Since the comparison mapping is linear, it has a matrix representation. The matrix is the Jacobian, and it can be viewed as consisting of $N$ ! column vectors with $N(N-1) / 2$ components. Each of these vectors corresponds to one of the N! rankings; the vector has component values of either 1 or -1 to reflect the direction of the pairwise 
comparisons. To show that the comparison mapping has maximal rank, it suffices to show that this set of $N$ ! vectors inciudes $N(N-1), 2$ linearly independent vectors. Corisider vectors $W_{j}, j=1, \ldots, N(N-1) / 2$, where $V_{j}$ has the value 1 in the first $\{N(N-1) / 2\}-(j-1)$ components and -1 in the remaining components. This set of vectors is lirearly independent. This is because they form a square array where all of the entries on and above the diagonal from the lower left hand corner to the upper right hand corner are all equal to 1 . All of the other entries equal -1 .

There are $2^{N}$ vectors with entries of either 1 or -1 . So, most of them are not related to the described ranking method. Thus, to complete the proof, it remains to show that each $U_{j}$ is associated with one of the N! rankings of the alternatives. The choice of the comporients and the vectors $U_{j}$ makes this fairly simple. The vector Wi corresponds to the ranking alyazl. Van. Vector $_{2}$ has -1 only irithat last component, and this corresponds to a transposition of an and an-1. Since these two alternatives are adjacent in the first ranking, the ranking for va can be

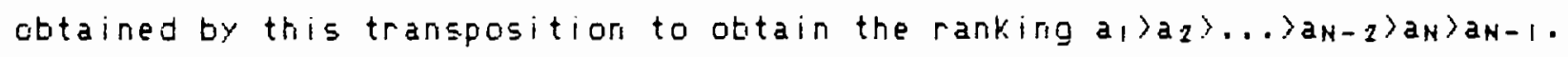
Indeed, the arly difference between $W_{j}$ and $V_{J+1}$ is in one component. This component reflects the change in the ranking in precisely one pair of two alternatives. Moreover, by construction, these two alternatives are adjacent in the ranking, RkJ, associated with Us. Therefore, the ranking for Usti is obtained by transposing these two alternatives in Fk. This is because the ordering of the choices of the pairs of alternatives to define the vectors is given in such a way that the - I's in the square array correspond to the $n$ adjacent transpositions required to move an from last place to first, then to move an-l fron what is now last place to second, etc. This completes the proaf.

Proof of Theorem 4 . Let the weight vectors $w_{k}, k=3, \ldots, N$ be as specified in the statement of the theorem. In this situation, each voter casts the weight of either 1 or - 1 for each of the pairs of alternatives in accordance with the scheme described 
above. Furthermore, the voter casts the appropriate permutation of the weight vector Wk to reflect the voter's ranking of the $k$ alternatives, $k=3, \ldots, N$. With the normalization described after the statement of Theorem 4 , this can be viewed as being

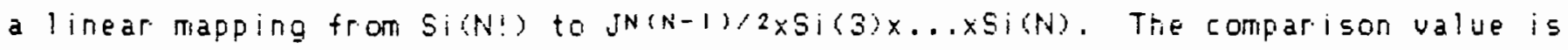
$\left(0,0, \ldots, 0 ;(1 / 3) E_{3}, \ldots,(1 / N) E_{N}\right)$. An interiar point which is mapped to this point is the same as the one given earlier; ramely, each of the N! possible rankings has the same number of voters.

Since the mapping is linear, its matrix representation defines the Jacobian. This matrix can be viewed as being $N$ ! column vectors with $(N(N-1) / 2\}+3+\ldots+i N=1 N 2-3$ components. SThe dimension of the image is smaller; it has dimensian N2-N-1. The difference results from the constraints defining the $N-2$ simplices $S i(k)$ in the image space.) The firet $N(N-1) / 2$ components of these vectars are described above. The next three components are some permutation of the componerits of Wa, etc. We must show that linear combinations can be made from this set of $N$ ! vectors to derive a set of $N^{2}-3$ vectors which are linearly independent. In the proof of the corollary, a set of $N(N-1) / 2$ vectors which are independent in the first $N(N-1) / 2$ components were determined. To obtain the remaining $(N(N+1) / 2\}-3$ indeperdent vectors, we take the vector assaciated with each of the N! rankings and add it to the vector associated with the reveral of this rarking. Each of the first N(N-1)/2 components of the vector associated with the reversed ranking will differ in sign from the original vector. Therefore, the sum vectors will have zeros in each of these first $N(N-1) / 2$ components. Consequently, these new vectors are or thagonal to the range space used in the proof of Corollary 4.4. Consequently, all that is required is to show that these riew vectors contain a set of $\{N(N+1) / 2\}-3$ independent vectors.

For a reversed neutral vector, these new vectors are all multiples of $E_{N}$. In all other cases, the ith component has the value $w_{1} w_{N}-1+1$. For example, for the weight vector $(4,3,0)$, the new vector corresponding to the rankings a $>b\rangle$ and $c\rangle t\rangle$ is $(4,6,4)$. The vector corresponding to $a\rangle c\rangle b$ and $b\rangle c\rangle$ a is $(4,4,6)$. In general, these 
new vectors would correspond to a weight vector except that they do not have the monotonicity to corresporid to either a monotone or an inverted weighted voting system. However, the results in [7] hald even if the weight vectors do not have one of these monotonicity properties. Therefore, the above reduces to a special case of [7], and this gives the required proof of independence.

Froof that a Borda weight vector will not work for $N=3$. Assume that the alternatives are $a, b$, and $c$. Assume that the Borda weight vector is $B_{3}=(3,2,1)$. The comparison mapping is linear and its image includes the comparison point $(0,0,0,6,6,6)$. (Because we didn't normalize $B_{z}$, the sum of the components of si(3) is 6.)

The comparison regions in $S i(3)$ are identified with the linear rankings of the three alternatives. To obtain them, note that if the axis of $R \exists$ are labelled in the usual $x, y, z$ notation, then the region $x>y$ corresponds to $a>b, y>z$ corresponds to $b>c$, $z>x$ corresponds to $c>a, e t c$. In this way, the simplex $S i(3)$ is divided into 6 open sets which are defined by the intersection of the simplex with the three hyperplaries $x=y, y=z$, and $z=x$. (See [6].)

Suppose that all of the different behavior described in the theorem could hold for $B_{3}$. This would mean that the image would have to meet each of the 6 regions of Si(3) as well as all of the open regions in $\mathrm{J3}^{3}$ in all, it would meet 48 open regions. If this happens, then, by the linearity of the mapping and a comparison of the dimensions of the domain and range, it follow that the mapping is onto a neighborhood of the comparison point, so the matrix has a maximal rank of 5 . We will show that this isn't possible by listing all six of the vectors and then extracting a four dimensional basis.

The vectors are: a $\rangle b\rangle c,(1,1,1 ; 3,2,1) ; a\rangle c\rangle b,(1,1,-1 ; 3,1,2) ; c\rangle a\rangle b$, $\langle 1,-1,-1 ; 2,1,3\rangle ; c\rangle b\rangle a,\langle-1,-1,-1 ; 1,2,3\rangle ; b\rangle c\rangle a,\langle-1,-1,1,1,3,2\rangle ;$ and $b\rangle a\rangle c$, $(-1,1,1 ; 2,3,1)$. However, these 6 vectors admit a basis consisting of the first three vectors and the vector $(0,0,0 ; 1,1,1)$. Thus, the system has corank 2 . Since this last 
vector is orthogonal to the image space, the system has corank 2 with respect to the image space. This, and the linearity of the mapping means that the comparison maping has a nonzero iritersection with 12 of the 48 admissible comparison regions. If the mapping were always consistent, then the mapping would meet only $3 !=6$ regions, Thus, the mapping still admits several "inconsistent" conclusions.

Extension of Theorem 4. The last part of the proof of Theorem 4 uses reference [?]. However this reference admits a wider variety of behavior. For example, at the level of $k$ alternatives, it admits $k-1$ weight vectors, WJk, which form, with Ek, a lirearly independent set. The theorem permits $k-1$ arbitrary rankings of the k alternatives, and then it asserts that there exist examples of voters so that when the same voters consider the set of $k$ alternatives, $k=3, \ldots, N$, by using the ith weight vector, then the outcome is the ith specified ranking of the alternatives. This is true for all choices of $i=1, \ldots, k-1$ and $k$.

A similar extension holds for Thearem 4 . Namely, for each k=3,..,N, choose weight vectors so that for some ranking, the new vectors cformed by adding the vector to the vector associated with the reversed ranking) and Ex are linearly independent. It is easy to see that for each $k$, there are k-1-[k/2] such vectors. Then, for each adopted weight vector, choose some ranking of the $k$ alternatives. The conclusion is that there exist examples of voters such that when the same voters use the ith weight vector to rank the $k$ alternatives, the outcome is the associated ranking. The conclusion concerning the pairs of alternatives remains the same. The proof of this statement is a straightfarward modificatian of the proof of Thearem 4.

Proof of Corollary 4.5. From Corollary 4.4, it follows that there is an open set of examples where the outcome in pairwise elections is the cycle al, a $2, a_{2}>a_{3}, .$, an-1>an, an>al. Consider the reversed cycle alian<an-1<..<az<al. To create an agenda where the alternative as will te selected, take the listing of 
the $N$ alternatives from this cycle which starts with the alternative immediately following as. For example, the agenda which will lead to as winning is az, $a_{1}, a_{N}, a_{N-1}, \ldots, a_{4}, a_{\Sigma}$.

The proof of the second part of this statement is nothing more than examining the boundary properties of $L-1(\underline{0})$ to find those examples which will define a cycle like the above one. An example manifesting this behavior and which will lead to the same cycle is one where an equal number of voters have each of the following preferences;

$\left.a_{1}>a_{2}>\ldots>a_{N} ; a_{2}>a_{3}>\ldots>a_{N}>a_{1} ; a_{3}>a_{4}\right\rangle \ldots a_{N}>a_{1}>a_{2}$. Note

that in each pairwise comparison, the winning alternative will receive either $2 / 3$ of the vote, or all of it! This is true for whichever agenda is used and whichever a) ternative wins.

The dice examele. This is a straightfarward computation. However, the domain point used in the inverse image of the comparison point should correspond to two identical dice, but where the value of the probability that a particular face will surface is left to the end of the computation. In other words, there are complications iri the computations with two fair dice where each face is equally likely to surface.

\section{Acknowledgements}

This research was supported by NSF Grant NSF IST-8111122, In addition, I am pleased to acknowledge conversations with $W$. W. Funkenbusch, Tom Miles, and Hans We inberger on these and on related topics. Sume of this work was done while I was wisiting the Institute for Mathematics and its Applications at the University of Minnesota. I would like to thank my hosts, Hans We inberger, George Sell, and Leo Hurwicz for their kind hospitality during my stay. 


\section{References}

1. C. Blyth, On Simpsan's paradox and the sure-thing principle, Jour. of Amer. Statistical Assoc. 67 (1972), PF 364-6

2. C. Blyth, Some probability paradoxes in choice from among rardom alternatives, Journal of the American Statistica) Association, 6? (1972) 366-73.

3. C. Li chen, The maximum and minimum probatilities of cyclic stochastic inequalities, Chinese Mathematics $2(1963), 279-283$.

4. P. Fishburn, Inverted orders for monatone scoring rules, Discrete Applied Mathematics $\underline{3}(1981), 27-36$.

5. R. Niemi and W. Riker, The choice of vating systems, Scientific American 234 SJune $1976), 21-27$.

6. D. Sari, Inconsistencies of weighted summation voting systems, Math of Operations Research $\geq(1982), 479-490$.

7. D. Saari, The ultimate of chaos in weighted voting systems, Northwestern University Center for Math Econ, Discussion paper 505, 1981, also, to appear in Aduances in Applied Mathematics.

8. D. Saari, The geometry of departmental politics, the scoring of track meets, and Arrow's Social choice theorem, Northwestern University preprint, October, 1978. 
9. H. Steinhaus and S. Trybula, On a paradox in applied probability, Bull. Acad. Polo Sci. I (1959), 67-69.

10. 2. Usiskin, Max-min probabilitities in the voting paradox, Annals of Mathematical Statistics, $35(1964), 857-62$.

11. C. Wagner, Simpson's paradox in real life, The American Statistician 36 (1982), $P p$ $46-48$. 